\title{
Observations on Orientation Relationships between Rutile and Brookite
}

M. Josefina Arellano-Jimenez ${ }^{1}$, Matthew T. Janish ${ }^{2}$, Weyshla A. Rodriguez-Rodriquez ${ }^{2}$, Bonnie B. McKenzie ${ }^{3}$, Joseph R. Michael ${ }^{3}$ and C. Barry Carter ${ }^{2,4}$

${ }^{1}$ Department of Physics and Astronomy. UTSA. One UTSA Circle, San Antonio, TX 78249

${ }^{2}$ Department of Materials Science and Engineering, University of Connecticut

${ }^{3}$ Sandia National Laboratories, Albuquerque, NM 87185-1405

${ }^{4}$ Department of Chemical and Biomolecular Engineering, University of Connecticut

$\mathrm{TiO}_{2}$ exists in nature in three different forms, namely rutile, anatase and brookite; it can also be prepared in the laboratory in each of these forms although the first two are by far the most common [1]. The transformation of anatase to rutile is well documented but that for brookite to rutile is not, in part because brookite is not often observed. It is common to find brookite and anatase together, where anatase is the major phase. These two phases could be easily taken for just one due to very similar diffraction patterns when analyzed by XRD. Anatase and brookite coexist at a consistent fraction until $600{ }^{\circ} \mathrm{C}$, after which the fraction of brookite will decrease. Above $1000{ }^{\circ} \mathrm{C}$ both phases completely transform into rutile. In this context, understanding the mechanisms of the transformation process, the effect of impurities, and their relationship with the crystal structure in $\mathrm{TiO}_{2}$ polymorphs becomes relevant for new developments.

Several sections of one naturally-grown single crystal of $\mathrm{TiO}_{2}$ were analyzed. Cross sections of the sample were cut and polished for SEM analysis. The materials have been examined in a Zeiss SEM equipped with EBSD and a JEOL TEM2010F equipment with a NanoMegas system.

Figure 1 shows a region of a natural crystal of Brookite that has partially transformed to Rutile. A small grain of $\mathrm{SiO}_{2}$ has been trapped at the interface between the two materials. Interesting features in this image include the presence of a large number of pores in one phase and the different contrast in recorded by the backscatter detector even though both materials are $\mathrm{TiO}_{2}$. The orientation relationships have been examined using both EBSD and precession diffraction.

References:

[1] C.B. Carter and M.G. Norton, Ceramic Materials: Science and Engineering, 2•d ed., Springer 2013.

[2] MJA-J acknowledges UTSA for use of the Microscopy Facility. MTJ and WAR-R acknowledge the Department of Education's GAANN fellowship program for research support and the Institute of Materials Science at the University of Connecticut for access to the JEOL 6335F FESEM and the FEI Strata 400S DualBeam FESEM FIB. Erica Pehmoeller, Jessica Bogart and Alice Kilgo are thanked for their assistance in specimen preparation. BBK and JRM acknowledge Sandia National Laboratories for support and access to facilities; Sandia National Laboratories is a multi-program laboratory managed and operated by Sandia Corporation, a wholly owned subsidiary of Lockheed Martin Corporation, for the U.S. Department of Energy's National Nuclear Security Administration under contract DE-AC04- 94AL85000. 


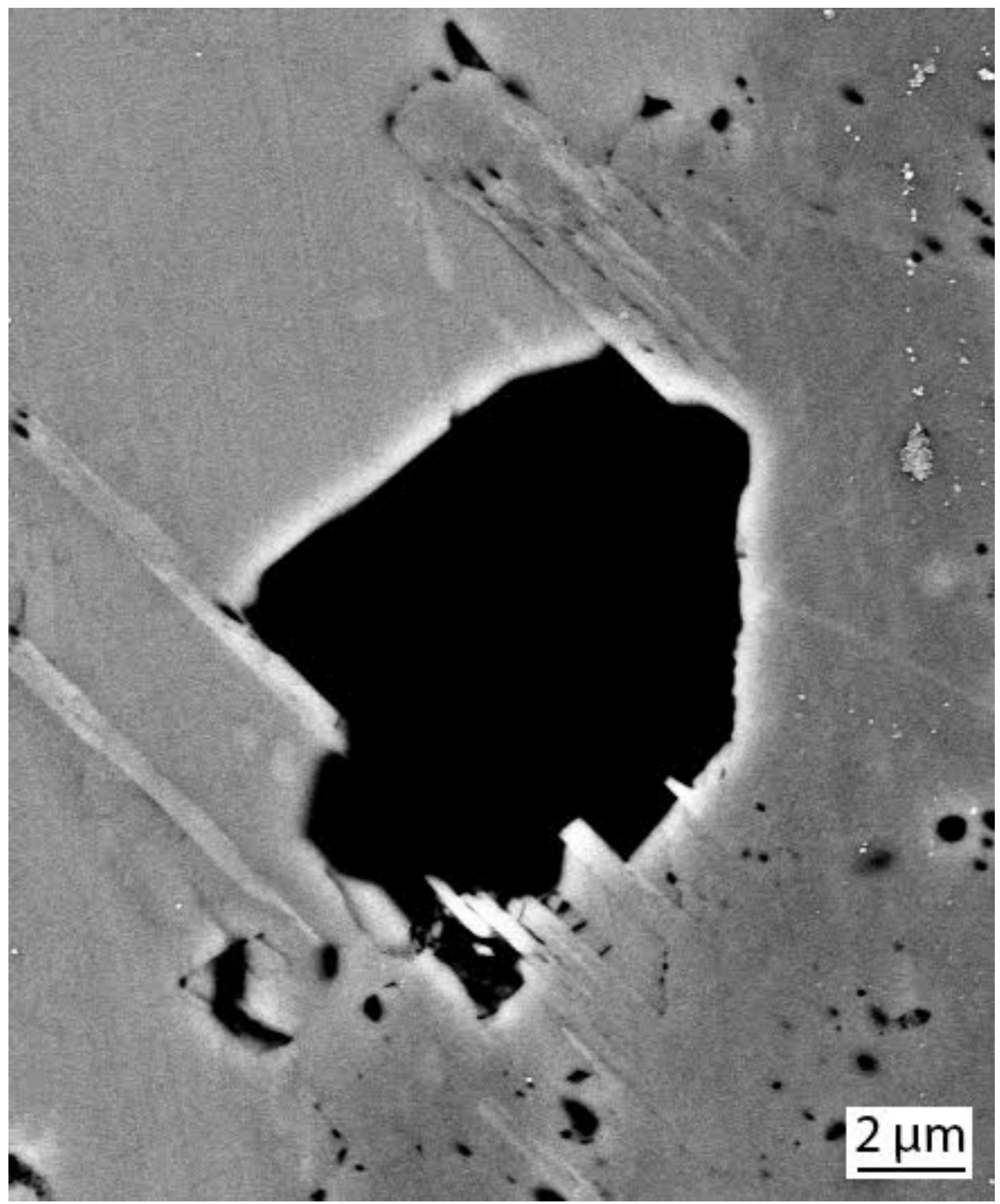

Figure 1. Natural crystal of brookite. Partial transformation from brookite to rutile and presence of $\mathrm{SiO}_{2}$ are observed. Some interesting features such as differences in porosity and BSE contrast between rutile and brookite polymorphs are also present. 